\title{
Comparative analysis of GC-MS of Isolona Engl. (Annonaceae) in Nigeria
} and the Cameroons

\author{
Sunday Adebunmi ADENIRAN ${ }^{1 *} \odot$, Akeem Babalola KADIRI ${ }^{2} \odot$, James Dele OLOWOKUDEJO ${ }^{3} \odot$ \\ ${ }^{1}$ Ilorin University, Department of Plant Biology, Ilorin, Nigeria \\ ${ }^{2,3}$ Lagos University, Department of Botany, Akoka Yaba Lagos, Nigeria \\ *adeniran.sa@unilorin.edu.ng, ${ }^{2}$ abkadiri2001@yahoo.com, ${ }^{3}$ olowokudejo@gmail.com
}

Received : 15.06 .2021
Accepted : 04.08 .2021

Nijerya ve Kamerun'daki Isolona Engl. (Annonaceae) bitkisinin
karşılaştırmalı GC-MS analizi

Abstract: The comparative assessment of six (6) Isolona species occurring in Nigeria and the Cameroons was undertaking using GC-MS analysis. The analysis was carried out with methanol extract and one hundred and seventy-six (176) phytoconstituents identified and scored at different retention times ranges from 3.22min in I. hexaloba (Pierre) Engl. \& Diels. to 34.56min in I. campanulata Engl. \& Diels.. The prominent compounds were scored in carboxylic and its derivates while the least compound was identified in alkyne. Highest M.wt. was 741.5 in I. congolana (De Wild.\&T.Durand) Engl. \& Diels. at retention time of $34.52 \mathrm{~min}$ and the lowest M.wt. was 84.15 in I. thonneri (De Wild.\&T.Durand) Engl. \& Diels. at retention time 3.44 min. Hexadecanoic acid, methyl ester formula $\mathrm{C}_{17} \mathrm{H}_{34} \mathrm{O}_{2}$ and M.wt of 270.4 was scored in all the six species suggested a diagnostic characters for the genus Isolona. Quantitatively, the amount of the analytes isolated in all the species ranges from $0.1 \%$ in I. hexaloba to $59.36 \%$ in I. thonneri. Two UPGMA distant trees were constructed with RMSD and Euclidean index to illustrate the relationships among the Isolona species based on the difference chemical constituents. The distance tree revealed the separation of I. thonneri from all other species, also a sub-cluster tree of I. hexaloba, I. campanulata and I. zenkeri (Engl.) Dyer. was also constructed. The highest distance value level is 9.576 scored between I. thonneri and I. congolana while the highest similarity level was 2.911 scored in I. hexaloba and I. campanulata. The overlapping phyto-constituents characters revealed the closeness of the taxa studied, this invariably will update the existing data in the genus and ultimately in the family Annonaceae.

Key words: Comparative analysis, GC-MS, Isolona, Phyto-constituents

Özet: Nijerya ve Kamerun'da bulunan altı (6) Isolona türünün GC-MS analizi kullanılarak karşılaştırmalı değerlendirmeleri yapılmıştır. Analiz metanol özütü ile gerçekleştirildi ve yüz yetmiş altı (176) fito-bileşen tanımlandı ve I heksaloba'da 3.22 dakika ile I. campanulata'da 34.56 dakika arasında değişen farklı alıkonma sürelerinde tanımlanmaları gerçekleştirildi. Öne çıkan bileşikler karboksilik ve türevleri olur iken, en az tepit edilen bileşikler alkinlerde tanımlandı. En yüksek M.wt. 34.52 dakikalık alıkonma zamanında $I$. congolana'da 741.5 olarak ölçülürken, düşük M.wt. değeri 84.15 ile 3.44 dk alıkonma süresinde I. thonneri'de tespit edildi. Heksadekanoik asit, metil ester formülü $\mathrm{C}_{17} \mathrm{H}_{34} \mathrm{O}_{2}$ ve $270.4 \mathrm{M}$.wt olarak değerlendirilen bileşik, altı türün tamamında tespit edildi ve Isolona cinsi için bir tanısal karakter önerildi. Kantitatif olarak, tüm türlerde izole edilen analit miktarı, I. hexaloba'da \%0.1 ile I. thonneri'de \%59.36'ya kadar değişim gösterdi. Farklı kimyasal bileşenlere dayalı olarak Isolona türleri arasındaki ilişkileri göstermek için RMSD ve Öklid indeksi ile iki UPGMA ağacı oluşturuldu. Oluşturulan ağaç, I. thonneri'nin diğer tüm türlerden ayrıldığını ortaya çıkarmış, ayrıca I. hexaloba, I. campanulata ve I. zenkeri'nin bir alt ağaç oluşturduğu görüldü. En yüksek uzaklık değeri I. thonneri ve I. congolana arasında 9.576 olarak puanır iken, en yüksek benzerlik düzeyi I. hexaloba ve I. campanulata'da 2.911 puan almıştır. Örtüşen fito-bileşen karakterleri, incelenen taksonların yakınlığını ortaya çıkardı, bu durum her cinsteki ve nihayetinde Annonaceae familyasındaki mevcut verileri güncellenmesine neden olacaktır.

Anahtar Kelimeler: Karşılaştırmalı analiz, GC-MS, Isolona, Fito-bileşenler

Citation: Adeniran SA, Kadiri AB, Olowokudejo JD (2021). Comparative analysis of GC-MS of Isolona Engl. (Annonaceae) in Nigeria and the Cameroons. Anatolian Journal of Botany 5(2): 102-111.

\section{Introduction}

Annonaceae Juss. are a pantropical family of trees, shrubs, and lianas belonging to the order Magnoliales (APGII, 2003) with $c .130$ genera and c. 2500 species (Chatrou et al., 2004). In Africa, the family comprises $c a .42$ genera and 500 species (Bremer et al., 2009; Smith et al., 2010; Couvreur et al., 2012; Zeng et al., 2014). The African genera were treated as a whole for the last time over 100 years ago by Engler and Diels (1901). Since then, regional accounts have been published in the second half of the last century such as those for Flore du Gabon (Le Thomas, 1969), Flora of West Tropical Africa (Hutchinson et al., 1954) and Flora of Tropical East Africa (Verdcourt, 1971). African Annonaceae have been largely understudied in recent years when compared to Neotropical and South-East Asian taxa (Maas et al., 2003; Couvreur et al., 2006; Couvreur et al., 2008). One of these genera is Isolona Engl., a sizeable genus with $\mathrm{ca}$. 21 species distributed across the tropical zone. Isolona Engl. flowers are hermaphroditic and are unusual as all six petals are basally connate, forming a single whorl (Couvreur, 2009). Relatively little is known about the reproductive biology, although the flowers of $I$. campanulata Engl. \& Diels have been shown to be protogynous, with the reproductive phases extending over a 2-day (or possibly 3-day) period, with diurnal receptivity (Gottsberger et al., 2011). The species are mainly trees and there are accounts on exomorphology (Maas et al., 2003; Couvreur, 2009). 
According to Panichpol and Waterman (1978), the Annonaceae is perhaps one of the least chemically known families'. A comprehensive review article published by" Leboeuf et al. (1982) updates all the important phytochemical research on Annonaceae members. Moreover, many earlier studies are only fragmentary and systematic reinvestigations are necessary in many cases. In order to draw valid conclusions on chemotaxonomic features of the Annonaceae, more chemical investigations are necessary. However, there is paucity of information on the structures of these chemicals and it has not been entrenched into taxonomic studies. Therefore, in search for additional identification criteria and pharmacological properties of the genus, the phyto-constituents characters of 6 African Isolona species occurring in Nigeria and Cameroons were investigated with a view to update the existing data within the family.

\section{Materials and Methods}

Dried herbarium samples of the six (6) species of Isolona from West and Central African obtained from the National Herbarium Yaounde, Cameroon YA(IH) were used for the study. The herbarium abbreviations follow Holmgren and Holmgren (2003) while the specific samples of the studied species and their herbarium information were Isolona campanulata: P.T. Francis, Dec. 1945; I. congolana: Westphal, 15/5/78; I. dewevrei: R. Letouvzey, 8/7/75; I. hexaloba: R. Letouvzey, 16/5/63; I. thonneri: R. Letouvzey, 23/3/70 and I. zenkeri: Endengle Elais, 19551956; showing species name, collector and date of collection respectively.

Two to $5 \mathrm{~g}$ of the plant sample was cut into small sizeable pieces and weighed then mixed with absolute methanol at a 1:20 ratio (100 $\mathrm{g}$ in $1 \mathrm{~L}$ solvent) and shaked thoroughly for 2-3 days. The extract was filtered by Whatman filter paper then the filtrate concentrated using the BUCHI Switzerland rotary evaporator to remove the methanol and obtain pure extract. $30 \mathrm{ml}$ of the solvent was repeated twice in the process and the extract was concentrated using nitrogen concentration and later treated with silica gel and anhydrous sodium sulphate. The GC-MS used for analysis is Agilent technologies 7890GC system coupled with mass spectrometer of model Agilent technologies 5975. The mobile phase is helium gas and the stationery phase is the column with length $30 \mathrm{~m}$, internal diameter $0.320 \mathrm{~mm}$, thickness $0.25 \mu \mathrm{m}$. The oven temperature program, initial temperature is $80{ }^{\circ} \mathrm{C}$ to hold for $2 \mathrm{~min}$ at $10{ }^{\circ} \mathrm{C}$ per min to final temperature of $240{ }^{\circ} \mathrm{C}$ to hold for 6 min, the volume injected $1 \mu \mathrm{l}$. Method follows Cunha et al. (2004) and Phrompittayarat et al. (2008).

The phytocomponents of the extracts from the different species were identified based on direct comparison with the database of National Institute Standard and Technology (NIST) and Royal Society of Chemistry and National Library of Medicine.

\subsection{Data analysis}

The separated compounds by GC-MS analysis were scored in binary matrices, where 0 stands for the absence and 1 stands for the presence of a compound for all studied Isolona species; these codes were detailed in Table 1. Statistical analysis of the data of the compounds identified was carried out by using an online program Dendro UPGMA (A dendrogram Construction Utility) using RMSD coefficient and a software Paleontological Statistics software used for constructing Euclidean similarity index, scatter plot, and PCA using used for the constructing. This method follows the approaches of Gamal et al. (2017).

\section{Results}

The GC-MS comparative analysis of six (6) species of Isolona namely: I. congolana, I. dewevrei, I. hexaloba, I. thonneri, I. zenkeri and I. campanulata isolated and identified one hundred seventy six (176) compounds at different retention times between $3.26 \mathrm{~min}$ and $34.56 \mathrm{~min}$ (Table 1). The highest number of compounds separated and recorded was in I. thonneri at retention time between 3.44 and $33.86 \mathrm{~min}$, while the lowest number of compounds was 29 at a retention time between 3.69 and $34.52 \mathrm{~min}$ in I. congolona. The highest M.wt. was 741.5 with a compound named Cyclodecasiloxane, eicosamethyl- and scored in I. congolana at retention time of $34.52 \mathrm{~min}$ and the lowest M.wt. was 78.13 (Mercaptoethanol, 2TMS derivatives) in I. thonneri at retention time $3.44 \mathrm{~min}$. The first compound dissociated in all the species was between $3.26 \mathrm{~min}$ and $3.69 \mathrm{~min}$ retention time except $I$. zenkeri at about $8.24 \mathrm{~min}$. The last compounds separated lies between retention time of 33.78 and $34.56 \mathrm{~min}$ but $I$. dewevrei last compound was dissociated at retention time of $20.39 \mathrm{~min}$. The amount of analytes isolated in all the species ranges from $0.1 \%$ with decided name: 1H-Indole-2-carboxylic acid, 6-(4ethoxyphenyl)-3-methyl-4-oxo-4,5,6,7-tetrahydro-,

isopropyl ester and hexasiloxane, 1,1,3,3,5,5,7,7,9,9, 11,11-dodecamethyl- in I. hexaloba to $59.36 \%$ with decided name: 1-Butanol, 2-methyl- ; cyclobutane, ethyland decane, 2,2,3-trimethyl- in I. thonneri. A compound named cyclononasiloxane, octadecamethyl was scored in I. congolana, I. zenkeri and I. campanulata. Also compounds with decided name octasiloxane, 1,1,3,3, $5,5,7,7,9,9,11,11,13,13, \quad 15,15$-hexadecamethyl- and heptasiloxane, $\quad 1,1,3,3, \quad 5,5,7,7,9,9,11,11,13,13$ tetradecamethyl- were scored in I. hexaloba, I. thonneri, I. zenkeri and I. campanulata. Likewise, hexadecanoic acid, methyl ester with the formula $\mathrm{C}_{17} \mathrm{H}_{34} \mathrm{O}_{2}$ and molecular weight of 270.4 was scored in all the six species (Table 1).

\subsection{Isolona congolana}

There were twenty-nine phyto-compounds identified in $I$. congolana broadly grouped into alkanes, alcohol, organocompound, alkaloid, carboxylic acids and its derivatives. A compound with decided name cyclodecasiloxane, eicosamethyl-, formula $\mathrm{C}_{20} \mathrm{H}_{60} \mathrm{O}_{10} \mathrm{Si}_{10}$ scored with the highest molecular weight of about 741.54 was found in $I$. congolana. Most prominent compounds in I. congolana were the carboxylic acids with fourteen (14) compounds and the least compound was an alkaloid with decided name benzene, nitroso- formula $\mathrm{C}_{6} \mathrm{H}_{5} \mathrm{NO}$ (Table 1). The retention time for the dissociation of the compounds was between 3.69 and $34.52 \mathrm{~min}$ with M.wt ranged from 107.11 in Benzene, nitroso- to 741.53 in cyclodecasiloxane, eicosamethyl- (Fig. 1).

\subsection{Isolona dewevrei}

The M.wt of thirty-two (32) compounds separated between 3.36 and 20.39 min retention times was between 92.19 in a compound with decided name trimethylsilyl fluoride formula $\mathrm{C}_{3} \mathrm{H}_{9} \mathrm{FSi}$ and 270.45 in a compound with 
Table 1. The GC-MS analysis of six (6) species of Isolona based on the phyto-chemical constituents

\begin{tabular}{|c|c|c|c|c|c|c|c|c|c|c|c|}
\hline $\mathbf{S} / \mathbf{N}$ & COMPOUNDS & R.T & Area \% & M.F & M.W & $\mathbf{A}$ & $\mathbf{B}$ & $\mathbf{C}$ & $\mathbf{D}$ & $\mathbf{E}$ & $\overline{\mathbf{F}}$ \\
\hline & ALCOHOL & & & & & & & & & & \\
\hline 1. & 1,2-Benzenediol, 3,5-bis(1,1-dimethylethyl)- & 32.86 & 4.13 & $\mathrm{C}_{14} \mathrm{H}_{22} \mathrm{O}_{2}$ & 222.32 & 0 & 0 & 0 & 0 & 0 & 1 \\
\hline 2. & 1-Butanol, 2-methyl- & 3.44 & 59.36 & $\mathrm{C}_{7} \mathrm{H}_{14} \mathrm{O}_{2}$ & 130.19 & 0 & 0 & 0 & 1 & 0 & 0 \\
\hline 3. & $\begin{array}{l}\text { 2-Naphthalenemethanol, decahydro-. alpha.,.alpha.,4a-trimethyl-8- } \\
\text { methylene-, [2R-(2.alpha.,4a.alpha.,8a.beta.)]- }\end{array}$ & 16.45 & 8.3 & $\mathrm{C}_{15} \mathrm{H}_{26} \mathrm{O}$ & 222.37 & 0 & 0 & 0 & 0 & 1 & 1 \\
\hline 4. & Benzenemethanol, 4-methyl- & 3.68 & 1 & $\mathrm{C}_{8} \mathrm{H}_{10} \mathrm{O}$ & 122.16 & 0 & 0 & 0 & 0 & 0 & 1 \\
\hline 5. & Benzyl alcohol & 7.384 & 7.81 & $\mathrm{C}_{7} \mathrm{H}_{8} \mathrm{O}$ & 108.14 & 0 & 1 & 0 & 0 & 0 & 0 \\
\hline 6. & Bicyclo[2.2.1]heptan-2-ol, 1,7,7-trimethyl-, acetate, (1S-endo)- & 11.258 & 5.88 & $\mathrm{C}_{12} \mathrm{H}_{20} \mathrm{O}_{2}$ & 196.29 & 1 & 0 & 0 & 0 & 0 & 0 \\
\hline 7 & Heptaethylene glycol, TBDMS & 17.21 & 1.62 & $\mathrm{C}_{20} \mathrm{H}_{44} \mathrm{O}_{8} \mathrm{Si}$ & 440.41 & 0 & 0 & 0 & 1 & 0 & 0 \\
\hline 8. & Mercaptoethanol, 2TMS derivative & 32.82 & 9.99 & $\mathrm{C}_{2} \mathrm{H}_{6} \mathrm{OS}$ & 78.13 & 0 & 0 & 0 & 0 & 1 & 0 \\
\hline 9. & Pentaethylene glycol, TBDMS & 17.21 & 1.62 & $\mathrm{C}_{16} \mathrm{H}_{36} \mathrm{O}_{6} \mathrm{Si}$ & 352.54 & 0 & 0 & 0 & 1 & 0 & 0 \\
\hline 10. & Phenol, 3,4-dimethyl- & 3.693 & 2.55 & $\mathrm{C}_{8} \mathrm{H}_{10} \mathrm{O}$ & 122.16 & 1 & 0 & 0 & 0 & 0 & 0 \\
\hline \multirow[t]{2}{*}{11.} & 1H-1,2,3-benzotriazole, 5,6-dichloro & 15.91 & 2.14 & $\mathrm{C}_{6} \mathrm{H}_{3} \mathrm{Cl}_{2} \mathrm{~N}_{3}$ & 188.01 & 0 & 1 & 0 & 0 & 0 & 0 \\
\hline & ALKALOID & & & & & & & & & & \\
\hline 12. & Benzene, nitroso- & 3.693 & 2.55 & $\mathrm{C}_{6} \mathrm{H}_{5} \mathrm{NO}$ & 107.11 & 1 & 0 & 0 & 0 & 0 & 0 \\
\hline 13. & Benzo[h]quinoline, 2,4-dimethyl- & 33.07 & 1.09 & $\mathrm{C}_{15} \mathrm{H}_{13} \mathrm{~N}$ & 207.27 & 0 & 0 & 1 & 1 & 0 & 1 \\
\hline 14. & Etiron & 3.67 & 0.28 & $\mathrm{C}_{3} \mathrm{H}_{9} \mathrm{BrN}_{2} \mathrm{~S}$ & 184.9 & 0 & 1 & 0 & 0 & 0 & 0 \\
\hline 15. & Hexestrol, 2TMS derivative & 33.86 & 0.24 & $\mathrm{C}_{24} \mathrm{H}_{38} \mathrm{O}_{2} \mathrm{Si}_{2}$ & 414.73 & 0 & 0 & 0 & 1 & 0 & 0 \\
\hline 16. & Oxime-, methoxy-phenyl-_ & 5.376 & 1.29 & $\mathrm{C}_{8} \mathrm{H}_{9} \mathrm{NO}_{2}$ & 151.16 & 0 & 0 & 1 & 0 & 0 & 0 \\
\hline 17. & 1-Methyl-2-aminomethylimidazole & 23.36 & 1.64 & $\mathrm{C}_{5} \mathrm{H}_{9} \mathrm{~N}_{3}$ & 111.15 & 0 & 0 & 0 & 1 & 0 & 0 \\
\hline 18. & 4-Benzamido-4-dichloromethyl-2-phenyl-2-imidazolin-5-one & 3.26 & 7.5 & $\mathrm{C}_{9} \mathrm{H}_{10} \mathrm{~N}_{2}$ & 146.19 & 0 & 0 & 0 & 0 & 0 & 1 \\
\hline 19. & Methenamine & 10.69 & 1.03 & $\mathrm{C}_{6} \mathrm{H}_{12} \mathrm{~N}_{4}$ & 140.19 & 0 & 0 & 1 & 0 & 0 & 0 \\
\hline 20 & 5-Methyl-2-phenylindolizine & 33.19 & 1.92 & $\mathrm{C}_{15} \mathrm{H}_{13}$ & 207.27 & 0 & 0 & 1 & 0 & 0 & 0 \\
\hline 21. & 1H-Benzo[4,5]furo[3,2-f]indole & 33.4 & 4.35 & $\mathrm{C}_{14} \mathrm{H}_{9} \mathrm{NO}$ & 207.23 & 0 & 0 & 0 & 1 & 0 & 0 \\
\hline 22. & 1H-Indole, 1-methyl-2-phenyl- & 32.78 & 0.8 & $\mathrm{C}_{15} \mathrm{H}_{13} \mathrm{~N}$ & 207.27 & 0 & 0 & 0 & 1 & 0 & 0 \\
\hline 23. & 1H-Indole, 5-methyl-2-phenyl- & 33.51 & 1.04 & $\mathrm{C}_{15} \mathrm{H}_{13} \mathrm{~N}$ & 207.27 & 0 & 0 & 0 & 1 & 0 & 0 \\
\hline 24. & 1H-Indole-2,3-dione, 5,7-dichloro- & 15.91 & 2.14 & $\mathrm{C}_{8} \mathrm{H}_{3} \mathrm{Cl}_{2} \mathrm{NO}_{2}$ & 216.02 & 0 & 1 & 0 & 0 & 0 & 0 \\
\hline 25. & $\begin{array}{l}\text { 1H-Indole-2-carboxylic acid, 6-(4-ethoxyphenyl)-3-methyl-4-oxo- } \\
\text { 4,5,6,7-tetrahydro-, isopropyl ester }\end{array}$ & 32.58 & 0.1 & $\mathrm{C}_{21} \mathrm{H}_{25}$ & 355.43 & 0 & 0 & 1 & 0 & 0 & 1 \\
\hline 26. & 3H-indole, 2-methyl-3-phenyl- & 33.76 & 1.83 & $\mathrm{C}_{16} \mathrm{H}_{15} \mathrm{~N}$ & 221.3 & 0 & 0 & 0 & 1 & 0 & 1 \\
\hline 27. & $\begin{array}{l}\text { 3-Amino-6-oxo-7a-phenyl-4aH,5H,7H-pyrrolo[2,3-c]pyridazine- } \\
\text { 4,5-dicarbonitrile }\end{array}$ & 3.68 & 1 & $\mathrm{C}_{12} \mathrm{H}_{10} \mathrm{~N}_{4} \mathrm{O}_{2}$ & 242.23 & 0 & 0 & 0 & 0 & 0 & 1 \\
\hline 28. & 2-Ethylacridine & 31.83 & 1.3 & $\mathrm{C}_{15} \mathrm{H}_{13} \mathrm{~N}$ & 207.27 & 0 & 0 & 1 & 1 & 0 & 1 \\
\hline 29. & $\mathrm{n}($ sup1),n(sup4)-dibenzoylpiperazin & 3.362 & 19.97 & $\mathrm{C}_{18} \mathrm{H}_{18} \mathrm{~N}_{2} \mathrm{O}_{2}$ & 294.3 & 0 & 0 & 1 & 0 & 0 & 0 \\
\hline 30. & N,N-Dimethyl-4-nitroso-3-(trimethylsilyl)aniline & 34.05 & 1.2 & $\mathrm{C}_{11} \mathrm{H}_{18} \mathrm{~N}_{2} \mathrm{OSi}$ & 222.36 & 0 & 0 & 0 & 0 & 0 & 1 \\
\hline 31. & Pyrido[2,3-d]pyrimidine, 4-phenyl- & 33.55 & 0.34 & $\mathrm{C}_{13} \mathrm{H}_{9} \mathrm{~N}_{3}$ & 207.23 & 0 & 0 & 1 & 0 & 0 & 0 \\
\hline 32. & 1-(4-Nitrophenyl)piperazine & 4.81 & 1.08 & $\mathrm{C}_{10} \mathrm{H}_{13} \mathrm{~N}_{3} \mathrm{O}_{2}$ & 207.23 & 0 & 0 & 0 & 1 & 0 & 0 \\
\hline \multirow[t]{2}{*}{33.} & .gamma.-Muurolene & 13.92 & 1.52 & $\mathrm{C}_{15} \mathrm{H}_{24}$ & 204.35 & 0 & 0 & 0 & 0 & 1 & 0 \\
\hline & ALKANE & & & & & & & & & & \\
\hline 34. & 2,3,5-Trioxabicyclo[2.1.0]pentane, 1,4-bis(phenylmethyl)- & 11.67 & 0.35 & $\mathrm{C}_{16} \mathrm{H}_{14} \mathrm{O}_{3}$ & 254.28 & 0 & 1 & 0 & 0 & 0 & 0 \\
\hline 35. & 1-Bromoeicosane & 8.359 & 2.29 & $\mathrm{C}_{20} \mathrm{H}_{41} \mathrm{Br}$ & 361.44 & 1 & 0 & 0 & 0 & 0 & 0 \\
\hline 36. & 2-Methylhexacosane & 8.519 & 2.98 & $\mathrm{C}_{27} \mathrm{H}_{56}$ & 380.7 & 1 & 0 & 0 & 0 & 0 & 0 \\
\hline 37. & Bicyclo[3.1.1]heptane, 2,6,6-trimethyl-, (1.alpha.,2.beta.,5.alpha.) & 19.05 & 2.27 & $\mathrm{C}_{10} \mathrm{H}_{18}$ & 138.25 & 0 & 0 & 1 & 0 & 0 & 0 \\
\hline 38. & Cyclobutane, ethyl- & 3.44 & 59.36 & $\mathrm{C}_{6} \mathrm{H}_{12}$ & 84.16 & 0 & 0 & 0 & 1 & 0 & 0 \\
\hline 39. & Decane, 2,2,3-trimethyl- & 3.44 & 59.36 & $\mathrm{C}_{13} \mathrm{H}_{28}$ & 184.36 & 0 & 0 & 0 & 1 & 0 & 0 \\
\hline 40. & Decane, 2-methyl- & 16.923 & 7.18 & $\mathrm{C}_{11} \mathrm{H}_{24}$ & 156.31 & 1 & 0 & 0 & 0 & 0 & 0 \\
\hline 41. & Dotriacontane & 16.831 & 2.24 & $\mathrm{C}_{32} \mathrm{H}_{66}$ & 450.87 & 1 & 0 & 0 & 0 & 0 & 0 \\
\hline 42. & Eicosane & 30.24 & 16.77 & $\mathrm{C}_{20} \mathrm{H}_{42}$ & 282.55 & 0 & 0 & 0 & 0 & 1 & 0 \\
\hline 43. & Eicosane, 9-octyl- & 32.49 & 11.79 & $\mathrm{C}_{28} \mathrm{H}_{58}$ & 394.76 & 0 & 0 & 0 & 0 & 1 & 0 \\
\hline 44. & Hentriacontane & 8.519 & 2.98 & $\mathrm{C}_{31} \mathrm{H}_{64}$ & 436.84 & 1 & 0 & 0 & 0 & 0 & 0 \\
\hline 45. & Heptacosane, 1-chloro- & 30.24 & 16.77 & $\mathrm{C}_{27} \mathrm{H}_{55} \mathrm{Cl}$ & 415.18 & 0 & 0 & 0 & 0 & 1 & 0 \\
\hline 46. & Hexadecane & 15.395 & 2.24 & $\mathrm{C}_{16} \mathrm{H}_{34}$ & 226.44 & 1 & 0 & 0 & 0 & 0 & 0 \\
\hline 47. & Hexadecane, 2,6,11,15-tetramethyl- & 16.923 & 7.18 & $\mathrm{C}_{20} \mathrm{H}_{42}$ & 282.5 & 1 & 0 & 0 & 0 & 0 & 0 \\
\hline 48. & Hexane, 2,2,3-trimethyl- & 3.361 & 0.98 & $\mathrm{C}_{9} \mathrm{H}_{20}$ & 128.25 & 0 & 1 & 0 & 0 & 0 & 0 \\
\hline 49. & Methylene chloride & 3.608 & 3.56 & $\mathrm{CH}_{2} \mathrm{Cl}_{2}$ & 84.93 & 0 & 0 & 1 & 0 & 0 & 0 \\
\hline 50. & Nonadecane & 32.49 & 11.79 & $\mathrm{C}_{18} \mathrm{H}_{38}$ & 254.49 & 0 & 0 & 0 & 0 & 1 & 0 \\
\hline 51. & Octacosane & 30.24 & 16.77 & $\mathrm{C}_{28} \mathrm{H}_{58}$ & 394.77 & 0 & 0 & 0 & 0 & 1 & 0 \\
\hline 52. & Pentane, 3-ethyl-2,2-dimethyl- & 3.361 & 0.98 & $\mathrm{C}_{9} \mathrm{H}_{20}$ & 128.25 & 0 & 1 & 0 & 0 & 0 & 0 \\
\hline 53. & Tetracontane, 3,5,24-trimethyl- & 15.395 & 2.24 & $\mathrm{C}_{43} \mathrm{H}_{88}$ & 605.2 & 1 & 0 & 0 & 0 & 0 & 0 \\
\hline \multirow[t]{2}{*}{54.} & Tridecane & 16.093 & 2.87 & $\mathrm{C}_{13} \mathrm{H}_{28}$ & 184.36 & 1 & 0 & 0 & 0 & 0 & 0 \\
\hline & ALKENE & & & & & & & & & & \\
\hline 55. & 5,7-Dimethylenebicyclo[2.2.2]oct-2 -ene & 11.67 & 0.35 & $\mathrm{C}_{10} \mathrm{H}_{12}$ & 132.2 & 0 & 1 & 0 & 0 & 0 & 0 \\
\hline 56. & $\begin{array}{l}\text { Bicyclo[7.2.0]undec-4-ene, 4,11,11-trimethyl-8-methylene-,[1R- } \\
\left.\left(1 \mathrm{R}^{*}, 4 \mathrm{Z}, 9 \mathrm{~S}^{*}\right)\right]_{-}\end{array}$ & 13.84 & 1.86 & $\mathrm{C}_{15} \mathrm{H}_{24}$ & 204.35 & 0 & 0 & 0 & 0 & 1 & 0 \\
\hline 57. & Neophytadiene & 19.05 & 2.27 & $\mathrm{C}_{20} \mathrm{H}_{38}$ & 278.51 & 0 & 0 & 1 & 0 & 0 & 0 \\
\hline 58. & Tricyclo[4.2.1.0(2,5)]non-7-ene, 3,4- & 32.69 & 0.64 & $\mathrm{C}_{27} \mathrm{H}_{64} \mathrm{O}_{6} \mathrm{Si}_{8}$ & 709.48 & 0 & 0 & 1 & 0 & 0 & 1 \\
\hline
\end{tabular}




\begin{tabular}{|c|c|c|c|c|c|c|c|c|c|c|c|}
\hline & hylsilyloxy)silyl)- & & & & & & & & & & \\
\hline 59. & $\begin{array}{l}\text { ALKYNE } \\
\text { 3-Octen-5-yne, (Z)- } \\
\text { BENZENE }\end{array}$ & 9.078 & 7.55 & $\underline{\mathrm{C}}_{8} \underline{\mathrm{H}}_{12}$ & 108.18 & 0 & 1 & 0 & 0 & 0 & 0 \\
\hline 60. & 1,3-Benzodioxole, 4-methoxy-6-(2-nitro-1-propenyl)- & 14.89 & 1.1 & $\mathrm{C}_{11} \mathrm{H}_{12} \mathrm{O}_{3}$ & 192.21 & 0 & 0 & 0 & 1 & 0 & 0 \\
\hline 61. & Benzene, 3-butenyl- & 11.67 & 0.35 & $\mathrm{C}_{10} \mathrm{H}_{12}$ & 132.20 & 0 & 1 & 0 & 0 & 0 & 0 \\
\hline 62. & $\begin{array}{l}\text { CARBOXYLIC AND ITS DERIVATIVES } \\
\text { 2'-Hydroxy-5'-methylacetophenone, TMS derivative }\end{array}$ & 33.57 & 0.4 & $\mathrm{C}_{9} \mathrm{H}_{10} \mathrm{O}_{2}$ & 150.17 & 0 & 0 & 1 & 0 & 0 & 0 \\
\hline 63. & N-Benzenesulfonylazetidin-3-one & 3.26 & 7.5 & $\mathrm{C}_{9} \mathrm{H}_{9} \mathrm{NO}_{3} \mathrm{~S}$ & 211.24 & 0 & 0 & 0 & 0 & 0 & 1 \\
\hline 64. & Propenone, 3-(2-benzoxazolylthio)-1-phenyl- & 12.67 & 0.93 & $\mathrm{C}_{16} \mathrm{H}_{11} \mathrm{NO}_{2} \mathrm{~S}$ & 280.99 & 0 & 0 & 0 & 1 & 0 & 0 \\
\hline 65. & 1,4-benzenedicarboxylic acid, mono(1-methylethyl) ester & 29.28 & 2.74 & $\mathrm{C}_{11} \mathrm{H}_{12} \mathrm{O}_{4}$ & 208.21 & 0 & 0 & 0 & 0 & 1 & 0 \\
\hline 66. & 10-Octadecenoic acid, methyl ester & 23.46 & 2.54 & $\mathrm{C}_{19} \mathrm{H}_{36} \mathrm{O}_{2}$ & 296.5 & 0 & 0 & 1 & 0 & 1 & 0 \\
\hline 67. & 12-Octadecenoic acid, methyl ester & 23.35 & 7 & $\mathrm{C}_{19} \mathrm{H}_{36} \mathrm{O}_{2}$ & 294.47 & 0 & 0 & 0 & 0 & 1 & 0 \\
\hline 68. & 13-Octadecenoic acid, methyl ester & 23.46 & 2.54 & $\mathrm{C}_{19} \mathrm{H}_{36} \mathrm{O}_{2}$ & 296.49 & 0 & 0 & 1 & 0 & 0 & 0 \\
\hline 69. & 14-Octadecenoic acid, methyl ester & 23.35 & 7 & $\mathrm{C}_{19} \mathrm{H}_{36} \mathrm{O}_{2}$ & 296.5 & 0 & 0 & 0 & 0 & 1 & 0 \\
\hline 70. & $\begin{array}{l}\text { 1-Benzazirene-1-carboxylic acid, 2,2,5a-trimethyl-1a-[3-oxo-1- } \\
\text { butenyl] perhydro-, methyl ester }\end{array}$ & 33.39 & 0.64 & $\mathrm{C}_{15} \mathrm{H}_{23} \mathrm{NO}_{3}$ & 265.35 & 0 & 0 & 1 & 1 & 0 & 0 \\
\hline 71. & 1-Butanol, 3-methyl-, acetate & 4.7 & 0.93 & $\mathrm{C}_{7} \mathrm{H}_{14} \mathrm{O}_{2}$ & 130.19 & 0 & 1 & 0 & 0 & 0 & 0 \\
\hline 72. & $\begin{array}{l}\text { 1H-Cyclopenta[1,3]cyclopropa[1,2]benzene, octahydro-7-methyl- } \\
\text { 3-methylene-4-(1-methylethyl)-, [3aS- } \\
\text { (3a.alpha.,3b.beta.,4.beta.,7.alpha.,7aS*)]- }\end{array}$ & 13.92 & 1.52 & $\mathrm{C}_{15} \mathrm{H}_{24}$ & 204.35 & 0 & 0 & 0 & 0 & 1 & 0 \\
\hline 73. & 2-(5-Phenyl-2-oxazolyl)benzoicaci & 3.68 & 1 & $\mathrm{C}_{16} \mathrm{H}_{11} \mathrm{NO}_{3}$ & 265.26 & 0 & 0 & 0 & 0 & 0 & 1 \\
\hline 74. & 2-(Acetoxymethyl)-3-(methoxycarbonyl)biphenylene & 33.04 & 0.56 & $\mathrm{C}_{17} \mathrm{H}_{14} \mathrm{O}_{4}$ & 281.99 & 0 & 0 & 1 & 1 & 0 & 1 \\
\hline 75. & 2-(Isobutoxycarbonyl)benzoic acid & 29.36 & 3.06 & $\mathrm{C}_{12} \mathrm{H}_{14} \mathrm{O}_{4}$ & 222.24 & 0 & 0 & 1 & 0 & 0 & 0 \\
\hline 76. & 2,4,6-Trimethylbenzoic acid, TMS derivatives & 31.909 & 30.76 & $\mathrm{C}_{13} \mathrm{H}_{20} \mathrm{O}_{2} \mathrm{Si}$ & 236.38 & 1 & 0 & 0 & 0 & 0 & 0 \\
\hline 77. & 2-Amino-5-methylbenzoic acid & 5.376 & 1.29 & $\mathrm{C}_{8} \mathrm{H}_{9} \mathrm{NO}_{2}$ & 151.16 & 0 & 0 & 1 & 0 & 0 & 0 \\
\hline 78. & 2-Amino-6-methylbenzoic acid & 5.376 & 1.29 & $\mathrm{C}_{8} \mathrm{H}_{9} \mathrm{NO}_{2}$ & 151.16 & 0 & 0 & 1 & 0 & 0 & 0 \\
\hline 79. & 3-(4-Hydroxyphenyl)propionitrile & 3.693 & 2.55 & $\mathrm{C}_{9} \mathrm{H}_{9} \mathrm{NO}$ & 147.17 & 1 & 0 & 0 & 0 & 0 & 0 \\
\hline 80. & 3-Chloropropionic acid, benzyl ester & 9.198 & 2.65 & $\mathrm{C}_{10} \mathrm{H}_{11} \mathrm{ClO}_{2}$ & 198.64 & 0 & 1 & 0 & 0 & 0 & 0 \\
\hline 81. & 3-Methyl-hexanoic acid & 3.361 & 0.98 & $\mathrm{C}_{7} \mathrm{H}_{14} \mathrm{O}_{2}$ & 130.19 & 0 & 1 & 0 & 0 & 0 & 0 \\
\hline 82. & 3-Quinolinecarboxylic acid, 6,8-difluoro-4-hydroxy-, ethyl ester & 32.74 & 0.19 & $\mathrm{C}_{12} \mathrm{H}_{9} \mathrm{~F}_{2} \mathrm{NO}_{3}$ & 253.2 & 0 & 0 & 1 & 1 & 0 & 1 \\
\hline 83. & 3-Trimethylsilyloxystearic acid, trimethylsilyl ester & 30.03 & 10.04 & $\mathrm{C}_{24} \mathrm{H}_{52} \mathrm{O}_{3} \mathrm{Si}_{2}$ & 444.8 & 0 & 0 & 0 & 0 & 1 & 0 \\
\hline 84. & 4-Benzyloxybenzoic acid & 19.772 & 0.58 & $\mathrm{C}_{14} \mathrm{H}_{12} \mathrm{O}_{3}$ & 228.24 & 0 & 1 & 0 & 0 & 0 & 0 \\
\hline 85. & $\begin{array}{l}\text { 4-pyridinecarboxylic acid, 3-cyano-2-hydroxy-6-methyl-5-nitro-, } \\
\text { methyl ester }\end{array}$ & 14.89 & 1.1 & $\mathrm{C}_{8} \mathrm{H}_{6} \mathrm{~N}_{2} \mathrm{O}_{2}$ & 162.15 & 0 & 0 & 0 & 1 & 0 & 0 \\
\hline 86. & 7-Hexadecenoic acid, methyl ester (Z)- & 23.36 & 1.64 & $\mathrm{C}_{17} \mathrm{H}_{32} \mathrm{O}_{2}$ & 268.4 & 0 & 0 & 0 & 1 & 0 & 0 \\
\hline 87. & 9,12-Octadecadiynoic acid, methylester & 13.84 & 1.86 & $\mathrm{C}_{19} \mathrm{H}_{34} \mathrm{O}_{2}$ & 294.47 & 0 & 0 & 0 & 0 & 1 & 0 \\
\hline 88. & Acetic acid, [4-(1,1-dimethylethyl)phenoxy]-, methyl ester & 32.59 & 2.11 & $\mathrm{C}_{13} \mathrm{H}_{18} \mathrm{O}_{3}$ & 221.99 & 0 & 0 & 0 & 0 & 0 & 1 \\
\hline 89. & Acetic acid, 1,7,7-trimethyl-bicyclo[2.2.1]hept-2-yl ester & 11.258 & 5.88 & $\mathrm{C}_{12} \mathrm{H}_{20} \mathrm{O}_{2}$ & 196.29 & 1 & 0 & 0 & 0 & 0 & 0 \\
\hline 90. & acetic acid, 2-[bis(methylthio)methylene]-1-phenylhydrazide & 32.74 & 0.19 & $\mathrm{C}_{6} \mathrm{H}_{6} \mathrm{~N}_{2} \mathrm{~S}_{2}$ & 170.26 & 0 & 0 & 1 & 0 & 0 & 0 \\
\hline 91. & Acetic acid, phenylmethyl ester & 9.347 & 45.54 & $\mathrm{C}_{9} \mathrm{H}_{10} \mathrm{O}_{2}$ & 150.18 & 0 & 1 & 0 & 0 & 0 & 0 \\
\hline 92. & Acridine-9-carbaldehyde & 33.86 & 0.24 & $\mathrm{C}_{14} \mathrm{H}_{9} \mathrm{NO}$ & 207.23 & 0 & 0 & 0 & 1 & 0 & 0 \\
\hline 93. & Allyl 2-ethyl butyrate & 7.899 & 0.45 & $\mathrm{C}_{9} \mathrm{H}_{16} \mathrm{O}_{2}$ & 156.22 & 0 & 1 & 0 & 0 & 0 & 0 \\
\hline 94. & Benzoic acid, 2-hydroxy-, phenylmethyl ester & 19.772 & 0.58 & $\mathrm{C}_{17} \mathrm{H}_{18} \mathrm{O}_{3}$ & 270.32 & 0 & 1 & 0 & 0 & 0 & 0 \\
\hline 95. & Benzoic acid, 2-methoxy-, methyl ester & 4.81 & 1.08 & $\mathrm{C}_{9} \mathrm{H}_{10} \mathrm{O}_{3}$ & 166.17 & 0 & 0 & 0 & 1 & 0 & 0 \\
\hline 96. & Benzoic acid, hydrazide & 8.25 & 2.22 & $\mathrm{C}_{7} \mathrm{H}_{8} \mathrm{~N}_{2} \mathrm{O}$ & 136.15 & 0 & 0 & 0 & 0 & 0 & 1 \\
\hline 97. & Benzoic acid, methyl ester & 8.27 & 0.33 & $\mathrm{C}_{8} \mathrm{H}_{8} \mathrm{O}_{2}$ & 136.15 & 0 & 0 & 0 & 1 & 1 & 1 \\
\hline 98. & Bis(2-ethylhexyl) phthalate & 29.36 & 3.06 & $\mathrm{C}_{24} \mathrm{H}_{38} \mathrm{O}_{4}$ & 390.56 & 0 & 0 & 1 & 1 & 1 & 0 \\
\hline 99. & Butanoic acid, ethyl ester & 3.459 & 3.21 & $\mathrm{C}_{6} \mathrm{H}_{12} \mathrm{O}_{2}$ & 116.16 & 0 & 1 & 0 & 0 & 0 & 0 \\
\hline 100. & Carbonic acid, prop-1-en-2-yl tridecyl ester & 16.093 & 2.87 & $\mathrm{C}_{17} \mathrm{H}_{32} \mathrm{O}_{3}$ & 284.43 & 1 & 0 & 0 & 0 & 0 & 0 \\
\hline 101. & Carbonic acid, undecyl vinyl ester & 16.831 & 2.24 & $\mathrm{C}_{14} \mathrm{H}_{26} \mathrm{O}_{3}$ & 242.35 & 1 & 0 & 0 & 0 & 0 & 0 \\
\hline 102. & cis-13-Octadecenoic acid & 23.46 & 2.54 & $\mathrm{C}_{19} \mathrm{H}_{36} \mathrm{O}_{2}$ & 296.5 & 0 & 0 & 1 & 0 & 0 & 0 \\
\hline 103. & Diethyl Phthalate & 15.52 & 17.62 & $\mathrm{C}_{12} \mathrm{H}_{14} \mathrm{O}_{4}$ & 222.24 & 0 & 1 & 0 & 0 & 0 & 0 \\
\hline 104. & Diisooctyl phthalate & 29.28 & 2.74 & $\mathrm{C}_{24} \mathrm{H}_{38} \mathrm{O}_{4}$ & 390.6 & 0 & 0 & 0 & 0 & 1 & 0 \\
\hline 105. & Dodecanoic acid, methyl ester & 14.44 & 1.02 & $\mathrm{C}_{13} \mathrm{H}_{26} \mathrm{O}_{2}$ & 214.34 & 0 & 0 & 1 & 0 & 0 & 0 \\
\hline 106. & Ethyl (2-hydroxyphenyl)acetate, TMS derivative & 19.73 & 1.88 & $\mathrm{C}_{10} \mathrm{H}_{12} \mathrm{O}_{3}$ & 180.2 & 0 & 0 & 0 & 1 & 0 & 0 \\
\hline 107. & $\begin{array}{l}\text { Formic acid, 1-(4,7-dihydro-2-methyl-7-oxopyrazolo[1,5- } \\
\text { a]pyrimidin-5-yl)-, methyl ester }\end{array}$ & 2.33 & 34.33 & $\mathrm{C}_{5} \mathrm{H}_{10} \mathrm{O}_{2}$ & 102.13 & 0 & 0 & 0 & 0 & 0 & 1 \\
\hline 108. & Heptadecanoic acid, 16-methyl-, methyl ester & 23.77 & 4.87 & $\mathrm{C}_{19} \mathrm{H}_{38} \mathrm{O}_{2}$ & 298.50 & 0 & 0 & 0 & 0 & 1 & 0 \\
\hline 109. & Heptanoic acid, methyl ester & 20.39 & 1.92 & $\mathrm{C}_{8} \mathrm{H}_{16} \mathrm{O}_{2}$ & 144.21 & 0 & 0 & 0 & 1 & 0 & 0 \\
\hline 110. & Hexadecanoic acid, 2-methyl- & 20.42 & 8.23 & $\mathrm{C}_{20} \mathrm{H}_{40} \mathrm{O}_{2}$ & 312.53 & 0 & 0 & 0 & 0 & 0 & 1 \\
\hline 111. & Hexadecanoic acid, methyl ester & 20.39 & 0.38 & $\mathrm{C}_{17} \mathrm{H}_{34} \mathrm{O}_{2}$ & 270.45 & 1 & 1 & 1 & 1 & 1 & 1 \\
\hline 112. & Hexanoic acid, 2-propenyl ester & 7.899 & 0.45 & $\mathrm{C}_{9} \mathrm{H}_{16} \mathrm{O}_{2}$ & 156.22 & 0 & 1 & 0 & 0 & 0 & 0 \\
\hline 113. & Isobornyl acetate & 11.258 & 5.88 & $\mathrm{C}_{12} \mathrm{H}_{20} \mathrm{O}_{2}$ & 196.29 & 1 & 0 & 0 & 0 & 0 & 0 \\
\hline 114. & Methoxyacetic acid, 2-pentadecyl ester & 8.359 & 2.29 & $\mathrm{C}_{18} \mathrm{H}_{36} \mathrm{O}_{3}$ & 300.5 & 1 & 0 & 0 & 0 & 0 & 0 \\
\hline 115. & Methoxyacetic acid, 2-tetradecyl ester & 8.359 & 2.29 & $\mathrm{C}_{17} \mathrm{H}_{34} \mathrm{O}_{3}$ & 286.4 & 1 & 0 & 0 & 0 & 0 & 0 \\
\hline 116. & Methoxyacetic acid, 3-tridecyl ester & 16.093 & 2.87 & $\mathrm{C}_{16} \mathrm{H}_{32} \mathrm{O}_{3}$ & 272.42 & 1 & 0 & 0 & 0 & 0 & 0 \\
\hline 117. & Methyl anthranilate & 12.05 & 3.05 & $\mathrm{C}_{8} \mathrm{H}_{9} \mathrm{NO}_{2}$ & 151.16 & 0 & 1 & 0 & 0 & 0 & 0 \\
\hline 118. & Methyl salicylate & 9.827 & 3.61 & $\mathrm{C}_{8} \mathrm{H}_{8} \mathrm{O}_{3}$ & 152.15 & 0 & 1 & 0 & 0 & 0 & 0 \\
\hline
\end{tabular}




\begin{tabular}{|c|c|c|c|c|c|c|c|c|c|c|c|}
\hline 119. & Methyl stearate & 23.86 & 2.53 & $\mathrm{C}_{19} \mathrm{H}_{38} \mathrm{O}_{2}$ & 298.50 & 0 & 0 & 1 & 0 & 1 & 1 \\
\hline 120. & Methyl tetradecanoate & 17.29 & 1.3 & $\mathrm{C}_{15} \mathrm{H}_{30} \mathrm{O}_{2}$ & 242.39 & 0 & 0 & 1 & 0 & 0 & 0 \\
\hline 121. & Methyl tropate, (.alpha.)-, TMS derivative & 19.73 & 1.88 & $\mathrm{C}_{10} \mathrm{H}_{12} \mathrm{O}_{3}$ & 180.20 & 0 & 0 & 0 & 1 & 0 & 0 \\
\hline 122. & N-Methyl-1-adamantaneacetamide & 32.54 & 0.45 & $\mathrm{C}_{13} \mathrm{H}_{21} \mathrm{NO}$ & 207.31 & 0 & 0 & 1 & 1 & 1 & 1 \\
\hline 123. & Octacosylheptafluorobutyrate & 16.831 & 2.24 & $\mathrm{C}_{32} \mathrm{H}_{57} \mathrm{~F}_{7} \mathrm{O}_{2}$ & 606.78 & 1 & 0 & 0 & 0 & 0 & 0 \\
\hline 124. & Pentadecanoic acid, 14-methyl-, methyl ester & 20.39 & 14.1 & $\mathrm{C}_{17} \mathrm{H}_{34} \mathrm{O}_{2}$ & 270.5 & 0 & 0 & 0 & 0 & 1 & 1 \\
\hline 125. & Pentadecanoic acid, methyl ester & 20.39 & 1.92 & $\mathrm{C}_{16} \mathrm{H}_{32} \mathrm{O}_{2}$ & 256.42 & 0 & 0 & 0 & 1 & 0 & 0 \\
\hline 126. & Perfluoropropionic acid, TBDMS derivate & 3.67 & 0.28 & $\mathrm{C}_{3} \mathrm{HF}_{5} \mathrm{O}_{2}$ & 164.03 & 0 & 1 & 0 & 0 & 0 & 1 \\
\hline 127. & Phthalic acid, heptyl oct-3-yl ester & 29.36 & 3.06 & $\mathrm{C}_{23} \mathrm{H}_{36} \mathrm{O}_{4}$ & 376.53 & 0 & 0 & 1 & 0 & 0 & 0 \\
\hline 128. & Prop-2-enoic acid, 2-cyano-3-(3-methyl-2-thienyl)-, methyl ester & 29.28 & 2.06 & $\mathrm{C}_{10} \mathrm{H}_{9} \mathrm{NO}_{2} \mathrm{~S}$ & 206.98 & 0 & 0 & 0 & 1 & 0 & 0 \\
\hline 129. & Silicic acid, diethyl bis(trimethylsilyl) ester & 33.1 & 1.91 & $\mathrm{C}_{10} \mathrm{H}_{28} \mathrm{O}_{4} \mathrm{Si}_{3}$ & 296.58 & 0 & 0 & 0 & 1 & 0 & 1 \\
\hline 130. & Sulfurous acid, butyl heptadecyl ester & 8.519 & 2.98 & $\mathrm{C}_{21} \mathrm{H}_{44} \mathrm{O}_{3} \mathrm{~S}$ & 376.6 & 1 & 0 & 0 & 0 & 0 & 0 \\
\hline 131. & Swep & 15.91 & 2.14 & $\mathrm{C}_{8} \mathrm{H}_{7} \mathrm{Cl}_{2} \mathrm{NO}_{2}$ & 219.98 & 0 & 1 & 0 & 0 & 0 & 0 \\
\hline 132. & Tetradecanoic acid, 12-methyl-, methyl ester & 20.436 & 4.71 & $\mathrm{C}_{16} \mathrm{H}_{32} \mathrm{O}_{2}$ & 256.42 & 1 & 0 & 0 & 0 & 0 & 1 \\
\hline 133. & Tridecanoic acid, 12-methyl-, methyl ester & 17.29 & 1.3 & $\mathrm{C}_{15} \mathrm{H}_{30} \mathrm{O}_{2}$ & 242.39 & 0 & 0 & 1 & 0 & 0 & 0 \\
\hline 134. & Trimethylsilyl 2-(trimethylsilyloxy)propaneperoxoate & 12.67 & 0.93 & $\mathrm{C}_{13} \mathrm{H}_{33} \mathrm{NO}_{3} \mathrm{Si}_{3}$ & 335.66 & 0 & 0 & 0 & 1 & 0 & 0 \\
\hline 135. & Undecanoic acid, 10-methyl-, methyl ester & 14.44 & 1.02 & $\mathrm{C}_{13} \mathrm{H}_{26} \mathrm{O}_{2}$ & 214.34 & 0 & 0 & 1 & 0 & 0 & 0 \\
\hline 136. & Benzylcarbamate & 9.347 & 45.54 & $\mathrm{C}_{8} \mathrm{H}_{9} \mathrm{NO}_{2}$ & 151.16 & 0 & 1 & 0 & 0 & 0 & 0 \\
\hline 137. & benzeneacetaldehyde, .alpha.-(methoxymethylene)-4-nitro- & 32.78 & 0.8 & $\mathrm{C}_{10} \mathrm{H}_{9} \mathrm{NO}_{4}$ & 207.18 & 0 & 0 & 0 & 1 & 0 & 0 \\
\hline 138. & Octanal, 2-(phenylmethylene)- & 17.74 & 0.92 & $\mathrm{C}_{15} \mathrm{H}_{20} \mathrm{O}$ & 216.32 & 0 & 1 & 0 & 0 & 0 & 0 \\
\hline 138. & $(2 \mathrm{E}, 4 \mathrm{E})-\mathrm{N}$-Isobutyltetradeca-2,4-dienamide & 33.24 & 0.32 & $\mathrm{C}_{18} \mathrm{H}_{33} \mathrm{NO}$ & 279.46 & 0 & 0 & 1 & 0 & 0 & 0 \\
\hline 139. & Benzamide, N-[6-(2-furyl)-2-oxo-2H-pyran-3-yl]- & 8.22 & 0.41 & $\mathrm{C}_{16} \mathrm{H}_{11} \mathrm{NO}_{4}$ & 256.99 & 0 & 0 & 0 & 1 & 0 & 0 \\
\hline 140. & Salicylamide & 9.827 & 3.61 & $\mathrm{C}_{7} \mathrm{H}_{7} \mathrm{NO}_{2}$ & 137.14 & 0 & 1 & 0 & 0 & 0 & 0 \\
\hline 141. & Silane, ethenyldiethylmethyl- & 23.67 & 1.79 & $\mathrm{C}_{7} \mathrm{H}_{16} \mathrm{Si}$ & 128.29 & 0 & 0 & 1 & 0 & 0 & 0 \\
\hline 142. & 1-(2-Thienyl)ethanonesemicarbazoneditms & 17.21 & 1.62 & $\mathrm{C}_{6} \mathrm{H}_{6} \mathrm{OS}$ & 126.18 & 0 & 0 & 0 & 1 & 0 & 0 \\
\hline 143. & $\begin{array}{l}\text { 1-(4-Methoxy-phenyl)-5,5-dioxo-hexahydro-5.lambda.(6)- } \\
\text { thieno[3,4-b]pyrrol-2-one }\end{array}$ & 29.28 & 2.06 & $\mathrm{C}_{13} \mathrm{H}_{15} \mathrm{NO}_{4} \mathrm{~S}$ & 281.33 & 0 & 0 & 0 & 1 & 0 & 0 \\
\hline 144. & 1,2,4-Triazine-3,5(2H,4H)-dione, 6-benzoylthio- & 8.22 & 0.41 & $\mathrm{C}_{7} \mathrm{H}_{11} \mathrm{~N}_{3} \mathrm{O}_{2}$ & 169.18 & 0 & 0 & 0 & 1 & 0 & 0 \\
\hline 145. & 2-(Ethyl)oxybenzylideneacetophenone & 33.61 & 0.58 & $\mathrm{C}_{17} \mathrm{H}_{16} \mathrm{O}_{2}$ & 252.31 & 0 & 0 & 0 & 0 & 0 & 1 \\
\hline 146. & 2-(n-Propyl)oxybenzylideneacetophenone & 33.55 & 0.34 & $\mathrm{C}_{5} \mathrm{H}_{12} \mathrm{O}_{2}$ & 104.15 & 0 & 0 & 1 & 1 & 0 & 1 \\
\hline 147. & 1H-Cycloprop[e]azulene, decahydro-1,1,7-trimethyl-4-methylene- & 13.84 & 1.86 & $\mathrm{C}_{15} \mathrm{H}_{24}$ & 204.35 & 0 & 0 & 0 & 0 & 1 & 0 \\
\hline 148. & Cryptomeridiol & 16.48 & 2.1 & $\mathrm{C}_{15} \mathrm{H}_{28} \mathrm{O}_{2}$ & 240.38 & 0 & 0 & 0 & 0 & 0 & 1 \\
\hline 149. & Phytol & 23.67 & 1.79 & $\mathrm{C}_{20} \mathrm{H}_{40} \mathrm{O}$ & 296.53 & 0 & 0 & 1 & 0 & 0 & 0 \\
\hline 150. & 2(3H)-Furanone, 5-hexyldihydro- & 15.18 & 1.3 & $\mathrm{C}_{11} \mathrm{H}_{20} \mathrm{O}_{2}$ & 184.28 & 0 & 1 & 0 & 0 & 0 & 0 \\
\hline \multirow[t]{2}{*}{151.} & delta.-Dodecalactone & 7.899 & 0.45 & $\mathrm{C}_{10} \mathrm{H}_{18} \mathrm{O}_{2}$ & 170.25 & 0 & 1 & 0 & 0 & 0 & 0 \\
\hline & NAPHTALENE & & & & & & & & & & \\
\hline 152. & 2-[4-Acetamidophenylsulfonyl]-1,4-naphthoquinone & 19.73 & 1.88 & $\mathrm{C}_{18} \mathrm{H}_{13} \mathrm{NO}_{5} \mathrm{~S}$ & 355.4 & 0 & 0 & 0 & 1 & 0 & 0 \\
\hline 153. & Anthracene, 9-ethyl-9,10-dihydro-9,10-dimethyl- & 33.46 & 0.84 & $\mathrm{C}_{18} \mathrm{H}_{20}$ & 236.4 & 0 & 0 & 0 & 1 & 0 & 0 \\
\hline 154. & $\begin{array}{l}\text { Naphthalene, 1,2,3,4,4a,5,6,8a-octahydro-7-methyl-4-methylene-1- } \\
\text { (1-m ethylethyl)-, (1.alpha.,4a.beta.,8a.alpha.)- }\end{array}$ & -13.92 & 1.52 & $\mathrm{C}_{15} \mathrm{H}_{24}$ & 204.35 & 0 & 0 & 0 & 0 & 1 & 0 \\
\hline \multirow[t]{2}{*}{155.} & Anthracene, 9,10-dihydro-9,9,10-trimethyl- & 32.73 & 0.29 & $\mathrm{C}_{17} \mathrm{H}_{18}$ & 222.32 & 0 & 0 & 1 & 0 & 0 & 0 \\
\hline & ORGANO-COMPOUNDS & & & & & & & & & & \\
\hline 156. & 1,1,1,3,5,5,5-Heptamethyltrisiloxane & 32.39 & 3.89 & $\mathrm{C}_{7} \mathrm{H}_{22} \mathrm{O}_{2} \mathrm{Si}_{3}$ & 222.51 & 0 & 0 & 0 & 0 & 0 & 1 \\
\hline 157. & 1,1,1,5,7,7,7-Heptamethyl-3,3-bis(trimethylsiloxy)tetrasiloxane & 34.523 & 36.3 & $\mathrm{C}_{13} \mathrm{H}_{39} \mathrm{O}_{5} \mathrm{Si}_{6}$ & 443.96 & 1 & 0 & 0 & 0 & 0 & 0 \\
\hline 158. & 3,3-Diisopropoxy-1,1,1,5,5,5-hexamethyltrisiloxane & 32.73 & 0.29 & $\mathrm{C}_{12} \mathrm{H}_{32} \mathrm{O}_{4} \mathrm{Si}_{3}$ & 324.63 & 0 & 0 & 1 & 1 & 0 & 1 \\
\hline 159. & Cyclodecasiloxane, eicosamethyl- & 34.523 & 36.3 & $\mathrm{C}_{20} \mathrm{H}_{60} \mathrm{O}_{10} \mathrm{Si}_{10}$ & 741.54 & 1 & 0 & 0 & 0 & 0 & 0 \\
\hline 160. & Cyclononasiloxane, octadecamethyl- & 32.82 & 9.99 & $\mathrm{C}_{18} \mathrm{H}_{54} \mathrm{O}_{9} \mathrm{Si}_{9}$ & 667.39 & 1 & 0 & 0 & 0 & 1 & 1 \\
\hline 161. & Heptasiloxane, 1,1,3,3,5,5,7,7,9,9,11,11,13,13-tetradecamethyl- & 33.86 & 0.91 & $\mathrm{C}_{14} \mathrm{H}_{42} \mathrm{O}_{6} \mathrm{Si}_{7}$ & 503.07 & 0 & 0 & 1 & 1 & 1 & 1 \\
\hline 162. & Hexasiloxane, 1,1,3,3,5,5,7,7,9,9,11,11-dodecamethyl- & 32.58 & 0.1 & $\mathrm{C}_{12} \mathrm{H}_{38} \mathrm{O}_{5} \mathrm{Si}_{6}$ & 430.94 & 0 & 0 & 1 & 1 & 0 & 0 \\
\hline 163. & Hexasiloxane, tetradecamethyl- & 31.909 & 30.76 & $\mathrm{C}_{14} \mathrm{H}_{42} \mathrm{O}_{5} \mathrm{Si}_{6}$ & 458.99 & 1 & 0 & 0 & 0 & 0 & 1 \\
\hline 164. & Methyltris(trimethylsiloxy)silane & 32.11 & 3.32 & $\mathrm{C}_{10} \mathrm{H}_{30} \mathrm{O}_{3} \mathrm{Si}_{4}$ & 310.69 & 0 & 0 & 1 & 0 & 0 & 1 \\
\hline 165. & $\begin{array}{l}\text { Octasiloxane, } 1,1,3,3,5,5,7,7,9,9,11,11,13,13,15,15- \\
\text { hexadecamethyl- }\end{array}$ & 32.54 & 0.72 & $\mathrm{C}_{16} \mathrm{H}_{48} \mathrm{O}_{7} \mathrm{Si}_{8}$ & 577.2 & 0 & 0 & 1 & 1 & 1 & 1 \\
\hline 166. & $\begin{array}{l}\text { Silane, [[5,5-dimethyl-4-methylene-2-(trimethylsilyl)-1- } \\
\text { cyclopenten-1-yl]methoxy]trimethyl- }\end{array}$ & 33.76 & 1.83 & $\mathrm{C}_{15} \mathrm{H}_{30} \mathrm{OSi}_{2}$ & 282.57 & 0 & 0 & 0 & 1 & 0 & 0 \\
\hline 167. & tert-Butyldimethylfluorosilane & 3.362 & 19.97 & $\mathrm{C}_{6} \mathrm{H}_{15} \mathrm{FSi}$ & 134.27 & 0 & 0 & 1 & 0 & 0 & 0 \\
\hline 168. & Tetrasiloxane, decamethyl- & 33.03 & 1.49 & $\mathrm{C}_{10} \mathrm{H}_{30} \mathrm{O}_{3} \mathrm{Si}_{4}$ & 310.69 & 0 & 0 & 0 & 1 & 0 & 0 \\
\hline 169. & Trimethylsilyl fluoride & 3.67 & 0.28 & $\mathrm{C}_{3} \mathrm{H}_{9} \mathrm{FSi}$ & 92.187 & 0 & 1 & 0 & 0 & 0 & 0 \\
\hline 170. & Trimethylsilyl-di(timethylsiloxy)-silane & 32.54 & 0.72 & $\mathrm{C}_{9} \mathrm{H}_{27} \mathrm{O}_{2} \mathrm{Si}_{4}$ & 279.65 & 0 & 0 & 0 & 1 & 0 & 0 \\
\hline 171. & Tris(tert-butyldimethylsilyloxy)arsane & 33.27 & 0.68 & $\mathrm{C}_{18} \mathrm{H}_{45} \mathrm{AsO}_{3} \mathrm{Si}_{3}$ & 468.7 & 0 & 0 & 1 & 0 & 0 & 0 \\
\hline 172 & 1-methyl-4-phenyl-5-thioxo-1,2,4-triazolidin-3-one & 34.27 & 2.7 & $\mathrm{C}_{9} \mathrm{H}_{9} \mathrm{~N}_{3} \mathrm{OS}$ & 207.25 & 0 & 0 & 0 & 0 & 0 & 1 \\
\hline 173. & 4-Methylphenol, n-propyl ether & 9.198 & 2.65 & $\mathrm{C}_{4} \mathrm{H}_{10} \mathrm{OC}_{10} \mathrm{H}_{14} \mathrm{O}$ & 150.22 & 0 & 1 & 0 & 0 & 0 & 0 \\
\hline 174. & 4-n-Hexylthiane, S,S-dioxide & 23.36 & 1.64 & $\mathrm{C}_{11} \mathrm{H}_{22} \mathrm{O}_{2} \mathrm{~S}$ & 218.36 & 0 & 0 & 0 & 1 & 0 & 0 \\
\hline 175. & Benzenecarbothioic acid & 8.22 & 0.41 & $\mathrm{C}_{7} \mathrm{H}_{6} \mathrm{OS}$ & 138.19 & 0 & 0 & 0 & 1 & 0 & 0 \\
\hline 176. & Benzoyl bromide & 8.24 & 3.89 & $\mathrm{C}_{7} \mathrm{H}_{5} \mathrm{BrO}$ & 185.02 & 0 & 0 & 0 & 0 & 1 & 0 \\
\hline
\end{tabular}

R.T- Retention time, P.A- Peak Area \%, M.F- Molecular formula, M.W- Molecular weight, A- Isolona congolana, B- I. dewevrei, C- I. hexaloba, D- I. thonneri, E- I. zenkeri, F- I.campanulata 


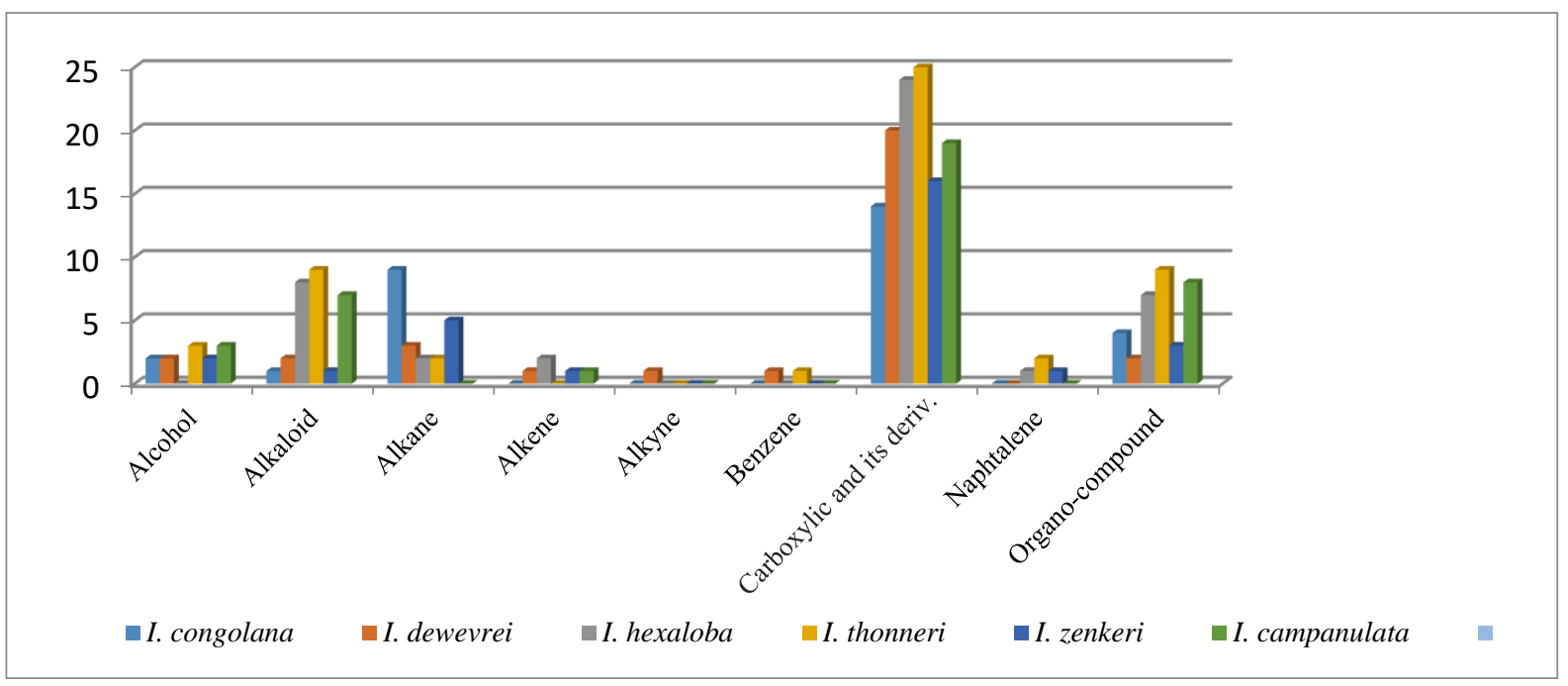

Figure 1: Showing the distribution of phyto-chemical compounds present in the Six (6) Isolona species

decided name hexadecanoic acid, methyl ester formula $\mathrm{C}_{17} \mathrm{H}_{34} \mathrm{O}_{2}$. The prominent group of compounds in these species were scored in carboxylic and its derivatives with about twenty (20) while the least compounds were scored in alkene, alkyne and benzen with the compounds named: 5,7-dimethylenebicyclo [2.2.2] oct-2 -ene; 3-octen-5-yne, (Z)- and benzene, 3-butenyl- respectively (Table 1). In this species, the amount of the analytes isolated ranges from $2.28 \%$ with etiron; perfluoropropionic acid, TBDMS derivates and trimethylsilyl fluoride to $45.54 \%$ with acetic acid, phenylmethyl ester and decided name: 1-butanol, 2methyl-; cyclobutane, ethyl- and benzylcarbamate (Table 1, Fig. 2).

\subsection{Isolona hexaloba}

In I. hexaloba, the retention time was between 3.36 and 33.78 min separated forty-four (44) compounds grouped into alkaloid, alkanes, alkenes, naphtalene, organocompounds, carboxylic acid and its derivatives with M.wt ranging from 84.9 in methylene chloride formula $\mathrm{CH}_{2} \mathrm{Cl}_{2}$ to 709.48 in Tricyclo[4.2.1.0 (2,5)]non-7-ene,3, 4di(tris(trimethylsilyloxy)silyl)- formula $\mathrm{C}_{27} \mathrm{H}_{64} \mathrm{O}_{6} \mathrm{Si}_{8}$. The following compounds: N-Methyl-1-adamantaneacetamide; Heptasiloxane, 1,1, 3,3,5,5,7,7,9,9,11,11,13, 13-tetradecamethyl- and Octasiloxane,1,1,3,3,5,5,7,7,9, $9,11,11,13,13,15,15$ - hexadecamethyl- were replicated in I. thonneri, I. zenkeri and I. Campanulata.

A compound with a decided named Methyl stearate formula $\mathrm{C}_{19} \mathrm{H}_{38} \mathrm{O}_{2}$ and M.wt of 298.50 was replicated in $I$. zenkeri and I. campanulata while Bis(2-ethylhexyl) phthalate formula $\mathrm{C}_{24} \mathrm{H}_{38} \mathrm{O}_{4}$ with M.wt 390.56 was scored in I. thonneri and I zenkeri. The most prominent were Carboxylic and its derivatives with about twenty-four (24) compounds (Table 1, Fig. 1).

\subsection{Isolona thonneri}

There are fifty-one (51) compounds identified in $I$. thonneri ranges from alcohol, alkaloid, alkanes, benzene, naphtalene, organo-compounds, carboxylic acids and its derivatives. The retention time for the dissociation of the compounds was between $3.44 \mathrm{~min}$ with 1-Butanol, 2methyl-; cyclobutane, ethyl-; and decane, 2,2,3-trimethylto 33.86 min with hexestrol, 2TMS derivative; acridine-9carbaldehyde and heptasiloxane, 1,1,3,3,5,5,7,7,9,9,11,11,13,13-tetradecamethyl-. Most prominent compounds were the carboxylic acid and its derivatives with twenty-five and least compound was scored in benzene with decided name 1,3-benzodioxole, 4methoxy-6-(2-nitro-1-propenyl)- formula $\mathrm{C}_{11} \mathrm{H}_{12} \mathrm{O}_{3}$ and W.mt 192.21. The following compounds namely: 2ethylacridine $\left(\mathrm{C}_{15} \mathrm{H}_{13} \mathrm{~N}\right)$; benzo[h]quinoline, 2,4-dimethyl$\left(\mathrm{C}_{15} \mathrm{H}_{13} \mathrm{~N}\right)$; 2-(Acetoxymethyl)-3-(methoxycarbonyl) biphenylene $\left(\mathrm{C}_{17} \mathrm{H}_{14} \mathrm{O}_{4}\right)$; 3-quinoline carboxylic acid, 6,8difluoro-4-hydroxy-, ethyl ester $\left(\mathrm{C}_{12} \mathrm{H}_{9} \mathrm{~F}_{2} \mathrm{NO}_{3}\right) ; 1,2,4$ Triazine-3,5(2H,4H)-dione, 6-benzoylthio- $\left(\mathrm{C}_{7} \mathrm{H}_{11} \mathrm{~N}_{3} \mathrm{O}_{2}\right)$ and 3,3-Diisopropoxy-1,1,1,5,5,5-hexamethyl trisiloxane $\left(\mathrm{C}_{12} \mathrm{H}_{32} \mathrm{O}_{4} \mathrm{Si}_{3}\right)$ were scored and duplicated in I. hexaloba and I. campanulata (Table 1, Fig. 1).

\subsection{Isolona zenkeri}

At the retention time $34.14 \mathrm{~min}$, thirty different (30) compounds were separated with major compounds in carboxylic and its derivative. The M.wt for these compounds ranged from 78.13 to 667.39 having sixteen (16) compounds of carboxylic and its derivatives, five (5) alkanes, two (2) alcohols and a compound each of alkaloid and alkene. A compound with M.wt136.15, formula $\mathrm{C}_{8} \mathrm{H}_{8} \mathrm{O}_{2}$ and decided name benzoic acid, methyl ester was scored in I. campanulata and I. thonneri. Also in this species, a compound with M.wt 667.39, formula $\mathrm{C}_{18} \mathrm{H}_{54} \mathrm{O}_{9} \mathrm{Si}_{9}$ and decided name cyclononasiloxane, octadecamethyl- was scored only in I. congolana and $I$. campanulata (Table 1, Fig. 1).

\subsection{Isolona campanualata}

At a retention time of $34.56 \mathrm{~min}$ thirty-eight (38) different compounds were separated and about 27 duplicate compound with decided name octasiloxane, $1,1,3,3,5,5,7,7,9,9,11,11,13,13,15,15$ -

hexadecamethyl-, formula $\mathrm{C}_{16} \mathrm{H}_{48} \mathrm{O}_{7} \mathrm{Si}_{8}$ and W.mt 577.2 at different quantity and retention time. The molecular weights for these compounds ranged from 104.15to 709.48 including fourteen (14) unique compounds (Table 1). Compound with M.wt667.39, formula $\mathrm{C}_{18} \mathrm{H}_{54} \mathrm{O}_{9} \mathrm{Si}_{9}$ and decided name cyclononasiloxane, octadecamethyl-was scored in I. congolana and I. zenkeri. A compound with decided name Methyl stearate, formula $\mathrm{C}_{19} \mathrm{H}_{38} \mathrm{O}_{2}$ and M.wt 
298.50 was scored with I. hexaloba and I. zenkeri. Two compounds name pentadecanoic acid, 14-methyl-, methyl ester and 2-naphthalenemethanol, decahydro- alpha., alpha., 4a-trimethyl-8-methylene-, [2R-(2.alpha., 4a.alpha., 8a.beta.)]- were both scored in I. zenkeri (Table 1, Fig. 2).

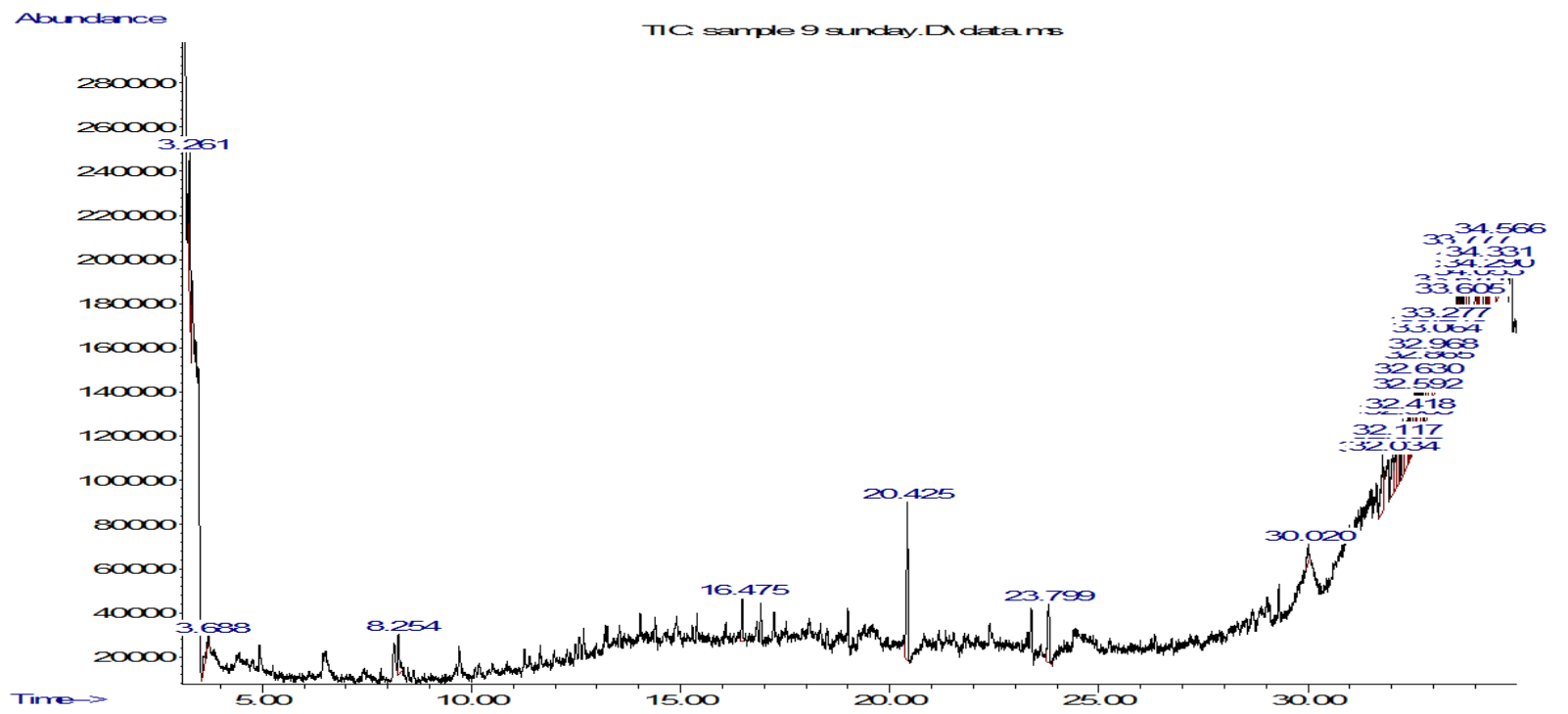

Figure 2. GC-MS Chromatogram of I. campanualata

\subsection{Systematic implications of GC-MS analysis on Isolona species relationship}

The distance values among the six (6) examined species showed evidence of similarity and differences using both the quantitative and qualitative phytochemical data. In general, the species varies from higher distance values to higher similarity (lower distance) values characterized by the species of the same genus indicated by the matrices (Figs. 4,5).

In a UPGMA distance tree based on the chemical constituents revealed by GC-MS between the six (6) Isolona species was presented. The distance matrix computed with Euclidean similarity index based on the quantitative characters of the constituents in each species (Fig. 4). The tree matrix produces clusters species and identified them to the specific. I. thonneri was well separated from all other species while the others were delineated within the sub-clusters. In figure 3 , it was also clear in the scatter plot that I. thonneri has the highest distance from the centre. To corroborate the tree, the highest distance value level (9.576) was scored between $I$. thonneri and I. congolana. This was represented in the clustering tree. On the other hands, the highest similarity level was 2.911 scored in I. hexaloba and I. campanulata. The sub-cluster of $I$. zenkeri, $I$. hexaloba and $I$. campanulata was clearly grouped together in the scatter plot and closer to the centre. The distance between $I$. zenkeri and I. hexaloba was 4.168, whereas the similarity level scored in I. campanulata and I. zenkeri was 3.615 (Fig. 3).

The relationship between the examined species based on the analysis qualitative characters of chemical constituents as indicated by the RMSD coefficient tree constructed was illustrated in Figure 4. As observed in the quantitative analysis (Fig. 4). I. thonneri was also efficiently separated from the other Isolona species (Figure 5). A sub-cluster of the tree produced three species namely $I$. hexaloba, $I$. campanulata and I. zenkeri from the remaining taxa.
Likewise in a cluster of I. dewevrei and I. congolana with genetic distance 5.367. The distance value between $I$. zenkeri and I. thonneri was 8.418 (Table 2).

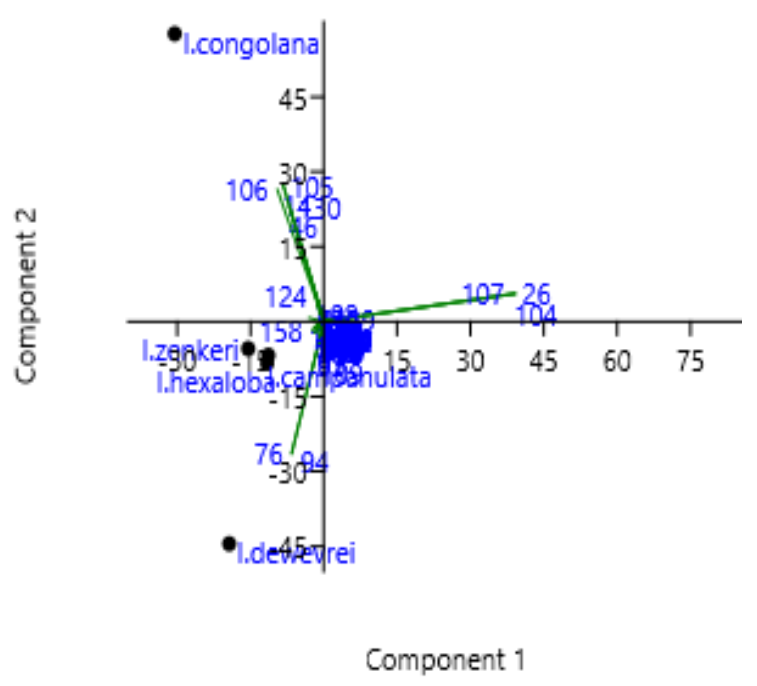

Figure 3. Scatter diagram based on the phyto-constituents of the six Isolona species

\section{Discussion}

Tetenyi (1987); Young et al. (1996); Akilan et al. (2014) and Gamal et al. (2017); have reported the systematics significance of phyto-constituents as an aid toward establishing the relationships among taxa below and above the species level. The GC-MS analysis separated all of the components in the examined samples and produced a specific spectral peak outputs. The total retention time to isolate the first compound and the last compound varies among the studied species. The differences in time taken for each chemical constituent to be dissociated are specific to the compounds and of great help in species identification. 
Table 2. Matrix computed based on the analysis of variation in chemical constituents by GC-MS analysis for the Isolona species

\begin{tabular}{|c|c|c|c|c|c|c|}
\hline & I. congolana & I. dewevrei & I. hexaloba & I. thonneri & I. zenkeri & I. campanulata \\
\hline I. congolana & 0 & 7.684 & 6.273 & 9.576 & 6.509 & 5.992 \\
\hline I. dewevrei & & 0 & 5.601 & 9.131 & 6.208 & 5.367 \\
\hline I. hexaloba & & & 0 & 7.990 & 4.168 & 2.911 \\
\hline I.thonneri & & & & 0 & 8.418 & 7.815 \\
\hline I. zenkeri & & & & & 0 & 3.615 \\
\hline I. campanulata & & & & & & 0 \\
\hline
\end{tabular}

Cardoso et al. (2008) opined on the important of secondary metabolite in taxonomic questions. The size of the analytes determines the amount of the specific constituent isolated which can be a diagnostic character. The clear differences in chemical formula, Molecular weight, time taken to dissociate all the compounds, amount of compound produced at different time, number and shapes of the compounds in each species were all considered to delineate the six (6) species of Isolona. Faustino et al. (2017) profiled four species of Calendula L. by categorizing the phyto-chemicals into major compounds. In this study, the hundred and seventy-six (176) compounds were grouped into alcohol, alkaloid, alkenes, alkynes, benzenes, carboxylic and its derivatives, naphthalene and organo-compounds for additional identification. The absence or presence of these phytoconstituents among the studied Isolona species were profiled and used in grading and scoring each species, this established the interrelationship between them. The abundance of these compounds in number and amount of analytes were important in chemotaxonomy delimitation of Isolona. In all the species, carboxylic and its derivative were the most profound among the hundred and seventysix compounds. It is noteworthy to mention that the present of Hexadecanoic acid, methyl ester was diagnostic for the genus Isolona having present in all the studied species.
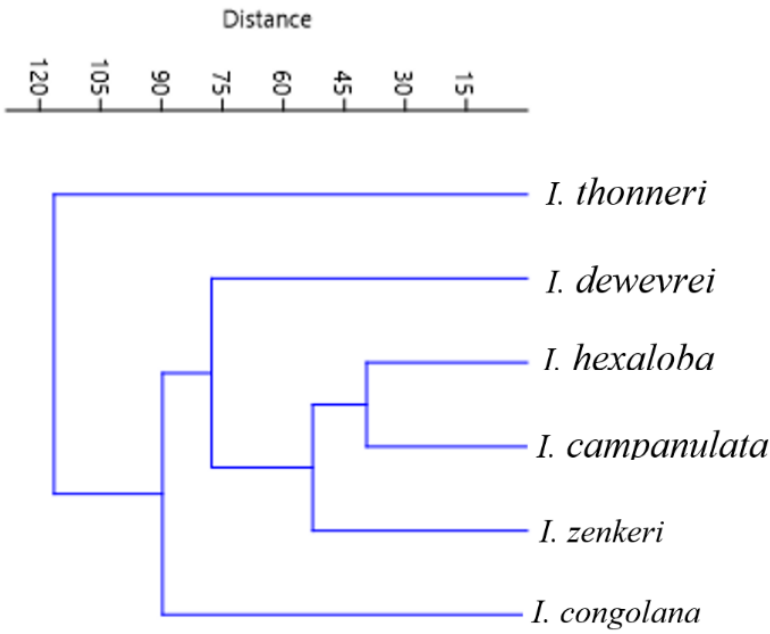

Figure 4. GC-MS analysis of UPGMA distant tree based on Euclidean similarity index showing the relationships among chemical constituents of Isolona species

Cardoso et al. (2008) and Mohy-UD-Din et al. (2010) investigated the phytoconstituents of the family Rubiaceae to analysis the taxonomic intricacies. The number of compounds produced was not the same in all the species studied. Graphical spectral peaks representation of each species revealed different phyto-compound at various retention times during dissociation. These were specific to the compound produced and scored. Gamal et al. (2017) categorized the compounds into six different retention times which was the basis for his work. In this 176 different compounds belonging to six (6) Isolona species were scored between 3.15 to $34.56 \mathrm{~min}$. All the species except $I$. zenkeri has average of about $3.35 \mathrm{~min}$ to produce the first compound. The number of phytoconstituents in different species contributed to the determination of the rank of each species. Likewise, the profound majority of the compounds to be an extraction of carboxylic and its derivatives of the compounds suggest the important of such compounds in the chemotaxonomy of the genus.

In the cluster analysis of plant chemical using the RMSD and Euclidean index showed I. thonneri was efficiently separated from the remaining species. The response of scatter plot is in support of the trees where it clustered the trio of I. zenkeri, I. hexaloba and I. campanulata. This suggests the closeness of the three species because they shared more phyto-constituents than the rest of the species. The highest and lowest distance values by the matrix affirmed the relatedness among the six (6) species studied in this research.

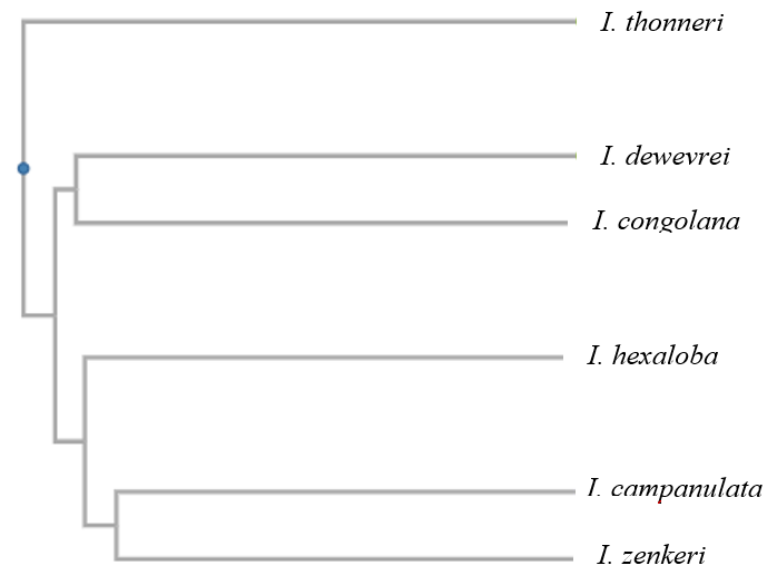

Figure 5. UPGMA distant tree constructed with RMSD coefficient showing the relationships among the Isolona species based on the qualitative analysis of chemical constituents

In conclusion, GC-MS analysis of methanol extract of six (6) Isolona species revealed one hundred and seventy-six (176) phytochemical constituents detected between 3.26 $\min$ to $34.56 \mathrm{~min}$. Twenty-nine (29), thirty-two (32), forty-four (44), fifty-one (51), thirty (30) and thirty-eight (38) phyto-constituents were dissociated from $I$. congolana, I. dewevrei, I. hexaloba, I. thonneri, I. zenkeri and I. campanulata. The highest M.wt. was 741.5 scored in $I$. congolana at retention time of $34.52 \mathrm{~min}$. and the lowest M.wt. was 84.15 in I. thonneri at retention 3.44 
min. Hexadecanoic acid, methyl ester formula $\mathrm{C}_{17} \mathrm{H}_{34} \mathrm{O}_{2}$ and M.wt of 270.4 was scored in all the six species studied. Similarity and distance matrices were calculated and two UPGMA distant trees constructed with RMSD and Euclidean similarity index to illustrate the relationships among the species based on the differences of chemical constituents. The overlapping phytoconstituents characters revealed the closeness of the taxa studied, this invariably will update the existing data in the genus and ultimately in the family.

\section{Conflict of Interest}

Authors have declared no conflict of interest.

\section{Authors' Contribution}

Kadiri AB and Olowokudejo JD contributed to manuscript development and literary work as supervisors of Adeniran SA who did data acquisition, writing and laboratory work.

\section{References}

Akilan CA, Srividhya M, Mohana Priya C, Samuel C, Sundara Mahalingam, MA (2014). Comparative analysis of phytochemicals, antibiogram of selected plants in Solanaceae family and its characterization studies. International Journal of Pharmacy and Pharmaceutical Sciences 6: 946-950.

Angiosperm Phylogeny Group II (2003). An update of the Angiosperm Phylogeny Group classification for the orders and families of flowering plants: APG II. Botanical Journal of the Linnean Society 141: 399-436.

Bremer B, Bremer K, Chase MW (2009). An update of the Angiosperm Phylogeny Group classification for the orders and families of flowering plants: APG III. Botanical Journal of the Linnean Society 161: 105-121.

Cardoso CL, Silva DHS, Young MCM, Castro G, Bolzani VS (2008). Indole monoterpene alkaloids from Chimarrhistur binata DC Prodr.: A contribution to the chemo-taxonomic studies of the Rubiaceae family. Brazilian Journal of Pharmacy18: 2629.

Chatrou LW, Koek-Noorman J, Maas PJM (2004). Annonaceae. In: Smith N, Morl SA, Henderson A, Stevenson DW, Heald SV (eds.). Flowering of Neotropics. Princeton: Princeton University Press, pp. 18-20.

Couvreur TLP, Gereau RE, Wieringa JJ, Richardson JE (2006). Description of four new species of Monodora and Isolona (Annonaceae) from Tanzania and an overview of Tanzanian Annonaceae diversity. Adansonia 28(2): 243-266.

Couvreur TLP (2009). Monograph of the syncarpous African genera Isolona and Monodora (Annonaceae). Systematic Botany Monographs 87: 1-150.

Couvreur TLP, Maas PJM, Meinke S, Johnson DM, Kebler PJA (2012). Keys to the genera of Annonaceae. Botanical Journal of the Linnean Society 169: 74-83.

Couvreur TLP, Richardson JE, Sosef MSM, Erkens RHJ, Chatrou LW (2008). Evolution of syncarpy and other morphological characters in African Annonaceae: a posterior mapping approach. Molecular Phylogenetics and Evolution 47: $302-318$.

Cunha IB, Sawaya AC, Caetano FM, Shimizu MT, Marcucci MC, Drezza FT, Povia GS, Carvalho PO (2004). Factors that influence the yield and composition Brazilian propolis extracts. Journal Brazil Chemical Society 15: 964-970.

Engler A, Diels L (1901). Annonaceae. In: Engler, A, (ed.). Monographien Afrikanischer pflanzen familien und gattungen VI Anonaceae. Leipzig: W. Engelmann, pp 1-96.

Faustino MV, Seca AML, Silveira P, Silva AMS, Pinto DC (2017). Gas chromatography-mass spectrometry profile of four Calendula L. taxa: A Industrial crops and products 104: 91-98.

Gamal AE, Soliman AH, Kamel S, Abdelfattah B (2017). Systematics implications of GC-MS analysis of secondary metabolites in the ethanol extract of Solanum species from South West Saudi Arabia. Egypt Journal of Botany 57(3): 429- 444.

Gottsberger G, Meinke S, Porembski S (2011). First records of flower biology and pollination in African Annonaceae: Isolona, Piptostigma, Uvariodendron, Monodora and Uvariopsis. Flora 206: 498-510.

Holmgren P, Holmgren N (2003). Index Herbariorum. Website: http://www.nybg.org/bsci/ih/ih.html / [accessed 31 May 2021].

Hutchinson J, Dalziel JM, Keay RWJ (1954). Flora of West Tropical Africa. Vol. 1, Part 1. London: Crown Agents for Oversea Governments and Administrations.

Le Thomas A (1969). Annonacées. Flore du Gabon, Vol. 16. Paris: Muséum National d'Histoire Naturelle.

Leboeuf M, Cavé A, Bhaumik PK, Mukherjee R (1982). The phytochemistry of the Annonaceae. Phytochemistry 21(12): 27832813.

Maas PJM, Westra LYT, Chatrou LW (2003). Duguetia. Flora Neotropica monograph 88. New York: The New York Botanical Garden.

Mohy-UD-Din A, Zaheer-UD-Din K, Ahmad M, Kashmiri MA (2010). Chemotaxonomic value of alkaloids in Solanum nigrum complex. Pakistan Journal of Botany 42: 653-660.

Panichpol K, Waterman PG (1978). Novel flavonoids from the stem of Popowia cauliflora. Phytochemistry 8(17): $1363-1367$.

Phrompittayarat W, Putalun W, Tanaka H, Jetiyanon K, Wittaya-areekul S, Ingkaninan K (2008). Comparison of various extraction methods of Bacopa monnier. Naresuan University Journal 15(1): 29-34.

Smith SA, Beaulieu JM, Donoghue MJ (2010). An uncorrelated relaxed-clock analysis suggests an earlier origin for flowering plants. Proceedings of the National Academy of Science of the United States of America107: 5897-5902.

Tetenyi P (1987). A chemotaxonomic classification of the Solanaceae. Annals of the Missouri Botanical Garden 74(3): 600-608. 
Adeniran et al. - Comparative analysis of...

Verdcourt B (1971). Annonaceae. In: Milne-Redhead E, PolhillR.M. (eds). Flora of Tropical East Africa. London: Crown Agents for Oversea Governments and Administrations, pp. 1-131.

Young MCM, Braga MR, Dietrich SMC, Bolzani VS, Trevisan LM, Gottlieb OR (1996). Chemosystematic markers of Rubiaceae. Opera Botanica Belgica 7: 205-212.

Zeng L, Zhang Q, Sun R, Kong H, Zhang N, Ma H. (2014). Resolution of deep angiosperm phylogeny using conserved nuclear genes and estimates of early divergence times. Nature Communications 5956: 1-12. 